\title{
El desarrollo de la matemática informal en los niños
}

\author{
Children's Informal Mathematical Development
}

\author{
Martín Malaspina Quevedo ${ }^{1}$ \\ Pontificia Universidad Católica del Perú
}

Recibido: $07-10$ - 17

Aceptado: $21-11-17$

\section{Resumen}

En el presente artículo se destaca la importancia del desarrollo del pensamiento matemático de los niños teniendo en cuenta que existen formas de aprender matemáticas que ocurren antes de vivir experiencias en la escuela, de una manera más natural, espontánea y recurriendo en gran medida a la observación y la imitación. Estos aprendizajes, llamados informales, tienen vital importancia para el adecuado aprendizaje formal de la matemática, que debe desarrollarse con base en ellos y teniendo en cuenta las emociones de los niños. Tales consideraciones deberían estar muy presentes en la formación inicial y continua de los profesores de educación infantil y así brindar mejores elementos para favorecer aprendizajes significativos y más agradables de la matemática en los niños.

Palabras clave: Pensamiento matemático; matemática informal; educación infantil; enseñanza y aprendizaje de la matemática.

\begin{abstract}
This article highlights the importance of the development of mathematical thinking of children taking into account that there are ways of learning mathematics that occur before living experiences in school, in a more natural, spontaneous way and using to a large extent the observation and the imitation. These learnings, called informal, are vitally important for the suitable formal learning of mathematics which must be developed based on them and taking into account the emotions of children. Such considerations should be very present in the initial and continuous training of pre school and primary teachers and thus provide better elements to encourage meaningful and more enjoyable children's mathematics learning.

Keywords: Mathematical thinking; informal mathematics; early childhood education; mathematics teaching and learning.
\end{abstract}

1 Docente Pontificia Universidad Católica del Perú. Email martin.malaspina@pucp.pe

(C) Los autores. Este artículo es publicado por la Revista de Investigación en Psicología de la Facultad de Psicología, Universidad Nacional Mayor de San Marcos. Este es un artículo de acceso abierto, distribuido bajo los términos de la licencia Creative Commons Atribucion - No Comercia_Compartir Igual 4.0 Internacional. (http://creativecommons.org/licenses/by-nc-sa/4.0/) que permite el uso no comercial, distribución y reproducción en cualquier medio, siempre que la obra original sea debidamente citada. 
"Los niños tienen su propia aritmética preescolar, que solo los psicólogos miopes podrían ignorar"

(Vygotsky, 1978, p. 84)

Cada vez hay un mayor consenso entre investigadores y especialistas, que trabajan en temas de desarrollo del niño y educación de la primera infancia, en que las experiencias de los niños durante sus primeros cinco años de vida influyen significativamente en muchos aspectos del desarrollo durante toda su vida (DeFlorio, 2011). Sin embargo, por un largo tiempo se ha pensado que no era oportuno hablar de la adquisición de conocimientos matemáticos en general durante la etapa infantil temprana (Alsina, 2006).

En contraste con la visión de que las matemáticas para los niños muy menores son inapropiadas para su desarrollo, investigaciones recientes han demostrado que los niños pequeños pueden pensar y entender contenidos matemáticos (Clements $\&$ Sarama, 2014). Actualmente, se sabe que el conocimiento matemático es un aspecto fundamental en el desarrollo infantil temprano (Baroody, Lai, \& Mix, 2006; Purpura, Baroody, \& Lonigan 2013).

Asimismo, se ha demostrado que el conocimiento matemático en esta etapa inicial de la vida proporciona una base significativa sobre la cual se construyen otras habilidades académicas. En relación a esto se ha encontrado que las habilidades matemáticas tempranas constituyen uno de los factores predictivos más fuertes de logros académicos a futuro (Duncan et al., 2007; Jordan, Kaplan, Ramineni, \& Locuniak, 2009; National Mathematics Advisory Panel, 2008). Desafortunadamente, los niños con conocimientos matemáticos tempranos más deficientes, por lo general continúan desarrollándose en matemáticas a un ritmo más lento que los compañeros más avanzados y es probable que permanezcan siempre con conocimientos matemáticos inferiores a los de ellos (Aunola, Leskinen, Lerkkanen, \& Nurmi, 2004).

Antes de que los niños comiencen sus experiencias en la escuela, existe un amplio rango de diferencias individuales en los conocimientos numéricos tempranos (Ramani \& Siegler, 2014). Este rango puede ser aún mayor en países como el Perú, por su diversidad social, económica, cultural y geográfica. La teoría y los estudios empíricos desde la perspectiva sociocultural sugieren que las experiencias de los niños pequeños en el entorno familiar y en contextos informales de aprendizaje del número pueden contribuir a explicar estas diferencias (Ramani \& Siegler, 2014). Teniendo en cuenta estas consideraciones, en el presente artículo se analiza cómo los contextos informales de aprendizaje juegan un papel esencial en el desarrollo de las habilidades matemáticas tempranas de los niños. 


\section{Aprendizaje informal en los niños}

Es común pensar que los principales aprendizajes de los niños se dan en las escuelas u otros contextos formales, en los cuales las enseñanzas se brindan de forma racionalizada y planificada. Sin embargo, existen formas de aprender que ocurren fuera de la escuela, de una manera más natural, espontánea y recurriendo en gran medida a la observación y la imitación; a este aprendizaje se le denomina informal (Paradise, 2017).

Para Piaget y Vygotsky los niños son exploradores activos de sus propios mundos, pero cabe resaltar que Vygotsky destaca en su teoría la importancia de las interacciones sociales para el desarrollo, dando gran énfasis al rol de la cultura y el ambiente social. Vygotsky (1988) adopta una aproximación sociocultural para explorar el desarrollo cognitivo de los niños y menciona que este desarrollo es el resultado de un proceso de aprendizaje de cómo usar los artefactos culturales (como el lenguaje y las matemáticas) con la interacción y apoyo de las personas que son mejores en el uso de estas herramientas, lo cual evidencia la relación de esta perspectiva con los aprendizajes informales de los niños.

Cabe resaltar que este tipo de educación informal se genera como parte integral de la vida cotidiana, en contextos familiares y comunitarios, y comprende comportamientos y procesos de aprendizaje que no suelen requerir planeación o intencionalidad, ni por parte del que aprende ni por el que apoya el aprendizaje (Paradise, 2017). También es importante destacar que el desarrollo de los personas es un proceso holístico y cada dimensión (biológica, cognitiva, emocional y social) influye y es influenciada por las otras (Crowley, 2017).

\section{Matemática informal en los niños}

La matemática es una parte inseparable de nuestra vida cotidiana y todas las personas la usan en diversos contextos y situaciones. La matemática ha sido investigada desde el siglo pasado por psicólogos y educadores desde diversas perspectivas y se han estudiado diversos aspectos del desarrollo de los conceptos y las habilidades matemáticas en niños e infantes, incluso se ha buscado el origen de las habilidades matemáticas humanas (Bryant \& Nuñes, 2002).

Al analizar los diversos estudios realizados sobre el desarrollo del pensamiento matemático en los niños, Ong (2016) menciona que muchas investigaciones de este tipo asumen que los niveles de desarrollo cognitivo por los que pasan los niños son universales y basan sus conclusiones en un análisis más individual del desarrollo de los niños, dejando al margen la importancia de la influencia sociocultural en el desarrollo del conocimiento y habilidades matemáticas de los niños. En este sentido, es importante resaltar que los niños participan en una variedad de actividades matemáticas en su vida cotidiana; sin embargo, dado que los objetivos 
y tipos de actividades matemáticas varían según las culturas, los niños participan en estas actividades en función a su propia valoración cultural (Guberman, 2004).

Por muchos años, varios psicólogos y educadores, influenciados por Piaget y su teoría del desarrollo cognitivo, no valoraron la investigación en el desarrollo matemático de los infantes y niños pequeños. Así, durante buen tiempo, muchas teorías del aprendizaje y del desarrollo cognitivo afirmaron que los niños pequeños carecen de conocimientos matemáticos antes de entrar en la escuela primaria (Starkey \& Klein, 2008). Sin embargo, ahora se sabe que las matemáticas que usan los niños durante la primera infancia son matemáticas intuitivas, y las aprenden en el marco de experiencias informales (Alsina, 2015); más aún, este uso de las matemáticas les permite explorar y dar un sentido al mundo (National Association of Young Children, 2008). En esta perspectiva, Ginsburg, Lee y Boyd (2008) afirman que la matemática cotidiana es un hecho esencial e incluso inevitable en el desarrollo cognitivo de los niños.

El desarrollo matemático de los niños suele ser similar al desarrollo histórico de las matemáticas: primero el conocimiento matemático es impreciso y concreto, pero luego va siendo más preciso y abstracto. De esta forma es cómo los preescolares van elaborando y perfeccionando el manejo de diversas técnicas con base en su matemática intuitiva. La matemática informal de los niños se va desarrollando a partir de necesidades básicas, prácticas y experiencias concretas (Baroody, 2000).

Losniños desde edades tempranas recopilan una gran riqueza de conocimientos sobre temas que les interesan y, a partir de estos intereses y actividades cotidianas, van desarrollando su pensamiento matemático. En sus actividades cotidianas, los niños pequeños participan en situaciones que promueven el desarrollo de habilidades, percepciones, estrategias, representaciones, actitudes, construcciones y operaciones relacionadas con una amplia gama de conceptos matemáticos (Nikiforidou, Pange, \& Chadjipadelis, 2013). En esta línea, Ginsburg et al. (2008) mencionan que los investigadores en los últimos años han acumulado evidencias que demuestran que, desde el nacimiento hasta los cinco años de edad, los niños desarrollan una matemática cotidiana (incluyendo ideas informales de más y menos, adición, sustracción, forma, tamaño, localización, patrones, posición, etc.) que es sorprendentemente amplia, compleja y a veces sofisticada.

Los niños pequeños suelen emplear su conocimiento informal como un medio para interpretar la matemática formal que se enseña en la escuela. Estas nociones matemáticas previas de los niños en el marco de un aprendizaje informal son el eslabón imprescindible para que luego puedan aprender las matemáticas en un contexto de enseñanza escolar que se caracteriza por una matemática escrita y simbólica (Baroody, 2000). 
Henningsten (2017) plantea que los niños llegan a la etapa preescolar con un entendimiento de las matemáticas y realiza un estudio reportando las historias que cuentan los niños y el uso de las matemáticas en estas historias, comprobando que en la mayoría de casos están presentes conceptos matemáticos profundos. Por otra parte, es evidente la presencia de emociones en los juegos de los niños y así, se perfila un reto importante para investigadores y educadores, que es el de optimizar el aprendizaje formal de contenidos matemáticos teniendo en cuenta tanto los aprendizajes informales previos como las emociones positivas que viven los niños en sus juegos y las emociones negativas que se generan al no tener en cuenta su propia matemática informal. Es pertinente tener en cuenta lo que nos dice Baroody (2000):

"Es frecuente que maestros, padres, compañeros, hermanos y otras pesonas hagan que los niños se sientan avergonzados de sus estrastegias informales. Como resultado de ello, los niños tratan de ocultar o disimular estas estrategias. Peor aún, empiezan a creer que sus métodcos informales no son válidos, que su manera de pensar en las matemáticas es inadecuada y estúpida” (p. 83)

\section{Reflexiones finales}

Es muy importante que los programas de formación inicial y continua de los profesores de educación infantil y de los primeros grados de primaria incluyan la toma de conciencia de aspectos muy importantes en el desarrollo cognitivo de los niños, como son los aprendizajes informales de la matemática que llevan a las aulas y las emociones positivas a estimular y las negativas a evitar.

Forzar el aprendizaje de la matemática poniendo énfasis en aspectos operativos o en el uso de reglas y símbolos que no entienden y sin encontrar relación con sus aprendizajes informales, llevará a los niños a la memorización y a un aprendizaje sin motivación, mecánico y desagradable de las matemáticas. Como lo sostienen Alsina (2015) y Baroody (2000) la conexión más importante en los primeros aprendizajes de la matemática es la existente entre las matemáticas intuitivas e informales y las matemáticas formales.

Otro aspecto a destacar es el importante rol que juega la familia como el entorno cotidiano más cercano en el cual el niño preescolar va desarrollando sus aprendizajes informales de la matemática. En ese sentido, psicólogos y educadores tenemos el reto de proponer intervenciones en las familias que favorezcan el desarrollo cognitivo en los niños, sobre todo teniendo en cuenta la diversidad sociocultural de nuestro país.

Finalmente, cabe destacar el rol importante de la psicología para brindar mejores elementos de entendimiento del pensamiento matemático informal de los niños, de sus emociones y de la importancia de los juegos en su desarrollo, a fin de estimular mejores aprendizajes de la matemática y actitudes positivas hacia ella. 


\section{REFERENCIAS}

Alsina, A. (2006). Cómo desarrollar el pensamiento matemático de 0 a 6 años. Barcelona: Octaedro.

Alsina, A. (2015). Matemáticas intuitivas e informales de 0 a 3 años. Elementos para empezar bien. Madrid: Narcea.

Aunola, K., Leskinen, E., Lerkkanen, M. K., \& Nurmi, J. E. (2004). Developmental dynamics of math performance from preschool to grade 2. Journal of Educational Psychology, 96(4), 699-713.

Baroody, A. (2000). El pensamiento matemático de los niños. Un marco evolutivo para maestros de preescolar, ciclo inicial y educación especial. Madrid: Visor.

Baroody, A., Lai, M., \& Mix, K. (2006). The development of young children's early number and operation sense and its implications for early childhood education. Handbook of Research on the Education of Young Children, 2, 187-221.

Bryant, P., \& Nuñes, T. (2002). Children's understanding of mathematics. En U. Goswami (Ed.), Blackwell handbook of childhood cognitive development (pp. 412-439). Malden, The Netherlands: Blackwell.

Clements, D. H., \& Sarama, J. (2014). Developing young children's mathematical thinking and understanding. En S. Robson \& S. Flannery (Eds.), The Routledge international handbook of young children's thinking and understanding (pp. 331-344). New York: Routledge.

Crowley, K. (2017). Child development: A practical introduction. London: Sage.

DeFlorio, L. (2011). The influence of the home learning environment on preschool children's informal mathematical development: Variation by age and socioeconomic status. University of California, Berkeley.

Duncan, G. J., Dowsett, C. J., Claessens, A., Magnuson, K., Huston, A. C., Klebanov, P., ... \& Japel, C. (2007). School readiness and later achievement. Developmental Psychology, 43(6), 1428-1446.

Ginsburg, H. P., Lee, J. S., \& Boyd, J. S. (2008). Mathematics education for young children: What it is and how to promote it. Society for Research in Child Development, 22(1), $1-23$.

Guberman, S. R. (2004). A comparative study of children's out-of-school activities and arithmetical achievements. Journal for Research in Mathematics Education, 35(2), 117-150.

Henningsten, M. (Setiembre, 2017). Young children's use of number and other mathematical ideas for their own purposes in inquiry-based learning environments. Trabajo presentado en Proceedings of the $14^{\text {th }}$ International Conference, Challenges in Mathematics Education for the Next Decade, Hungary.

Jordan, N. C., Kaplan, D., Ramineni, C., \& Locuniak, M. N. (2009). Early math matters: Kindergarten number competence and later mathematics outcomes. Developmental Psychology, 45, 850 - 867. doi:10.1037/a0014939 
National Association of Young Children. (2008). Early childhood mathematics: Promoting good beginnings. Washington, D.C.

National Mathematics Advisory Panel. (2008). Foundations for success: The final report of the National Mathematics Advisory Panel. Washington, DC: U. S. Department of Education.

Nikiforidou, Z., Pange, J., \& Chadjipadelis, T. (2013). Intuitive and informal knowledge in preschoolers' development of probabilistic thinking. International Journal of Early Childhood, 45(3), 347-357.

Ong, M. Y. L. (2016). Young children and mathematics: A relook at mathematical development from sociocultural perspectives. Bulletin of Faculty of Education, Hokkaido University, 126, 43-74.

Paradise, R. (2017). ¿Cómo educan los indígenas a sus hijos? El cómo y el por qué del aprendizaje en la familia y en la comunidad. En S. Frisancho, M. Moreno, P. Ruiz \& V. Zavala (Eds.), Aprendizaje, cultura y desarrollo, Una aproximación interdisciplinaria (pp. 41-58). Lima, Perú: Fondo Editorial PUCP.

Purpura, D. J., Baroody, A. J., \& Lonigan, C. J. (2013). The transition from informal to formal mathematical knowledge: Mediation by numeral knowledge. Journal of

Educational Psychology, 105(2), 453.

Ramani, G. B., \& Siegler, R. S. (2014). How informal learning activities can promote children's numerical knowledge. Oxford Handbooks Online. doi: 10.1093/ oxfordhb/9780199642342.013.012.

Starkey, P., \& Klein, A. (2008). Sociocultural influences on young children's mathematical knowledge. En O. Saracho \& B. Spodek (Eds.), Contemporary Perspectives on Mathematics in Early Childhood Education (pp. 253-276). Charlotte, NC: IAP.

Vygotsky, L. (1978). Mind in society: The development of higher psychological processes. Cambridge, MA: Harvard University Press.

Vygotsky, L. (1988). Pensamiento y lenguaje. Comentarios críticos de Jean Piaget. La Pléyade: Buenos Aires. 